\title{
Effect of hydrotherapy on the signs and symptoms of delayed onset muscle soreness
}

\author{
Joanna Vaile $\cdot$ Shona Halson • Nicholas Gill \\ Brian Dawson
}

Published online: 3 January 2008

(C) Springer-Verlag 2007

\section{Erratum to: Eur J Appl Physiol}

DOI 10.1007/s00421-007-0605-6

As a result of printing errors, several aspects of Table 1 (below) were incorrectly presented in the published version. There were formatting errors on columns 4 (i.e. CWI) and 6 (i.e. HWI). In addition, column 6 (HWI), row 6 should read 3,593 \pm 409 . The corrected version is shown below. The legend was correct.

The online version of the original article can be found under doi: 10.1007/s00421-007-0605-6.

J. Vaile $(\bowtie) \cdot$ S. Halson

Department of Physiology, Australian Institute of Sport,

PO Box 176, Belconnen, ACT, Australia

e-mail: jo.vaile@ausport.gov.au

N. Gill

School of Sport and Exercise Science,

Waikato Institute of Technology, Hamilton, New Zealand

N. Gill

Division of Sport and Recreation,

Auckland University of Technology,

Auckland, New Zealand

B. Dawson

School of Human Movement and Exercise Science,

University of Western Australia, Perth, Australia 
Table 1 Descriptive statistics (mean \pm SD) for dependent variables for each intervention and its independent control (CWT vs. PAS, CWI vs. PAS, and HWI vs. PAS)

\begin{tabular}{|c|c|c|c|c|c|c|}
\hline Variable & CWT & PAS & CWI & PAS & HWI & PAS \\
\hline \multicolumn{7}{|c|}{ Squat jump (peak power W) } \\
\hline Baseline & $3,938 \pm 871$ & $3,969 \pm 879$ & $4,158 \pm 945$ & $4,170 \pm 947$ & $3,902 \pm 303$ & $3,900 \pm 277$ \\
\hline $0 \mathrm{~h}$ post ex & $3,328 \pm 806$ & $3,479 \pm 792$ & $3,547 \pm 1,033$ & $3,564 \pm 878$ & $3,446 \pm 351$ & $3,382 \pm 278$ \\
\hline $24 \mathrm{~h}$ post ex & $3,675 \pm 741 *$ & $3,389 \pm 750$ & $3,735 \pm 872$ & $3,577 \pm 878$ & $3,459 \pm 389$ & $3,401 \pm 416$ \\
\hline $48 \mathrm{~h}$ post ex & $3,805 \pm 821 *$ & $3,473 \pm 755$ & $3,939 \pm 877^{*}$ & $3,507 \pm 795$ & $3,487 \pm 455$ & $3,460 \pm 370$ \\
\hline $72 \mathrm{~h}$ post ex & $3,937 \pm 808^{*}$ & $3,659 \pm 795$ & $4,080 \pm 914^{*}$ & $3,857 \pm 846$ & $3,593 \pm 409$ & $3,606 \pm 356$ \\
\hline \multicolumn{7}{|c|}{ Isometric squat (peak force $\mathrm{N}$ ) } \\
\hline Baseline & $2,068 \pm 446$ & $2,066 \pm 469$ & $2,110 \pm 472$ & $2,089 \pm 443$ & $1,929 \pm 295$ & $1,916 \pm 350$ \\
\hline $0 \mathrm{~h}$ post ex & $1,733 \pm 320$ & $1,750 \pm 389$ & $1,748 \pm 424$ & $1,734 \pm 420$ & $1,592 \pm 262$ & $1,597 \pm 271$ \\
\hline $24 \mathrm{~h}$ post ex & $1,857 \pm 405^{*}$ & $1,711 \pm 396$ & $1,877 \pm 418$ & $1,792 \pm 401$ & $1,685 \pm 286^{*}$ & $1,598 \pm 342$ \\
\hline $48 \mathrm{~h}$ post ex & $1,923 \pm 457^{*}$ & $1,783 \pm 424$ & $2,077 \pm 465^{*}$ & $1,769 \pm 412$ & $1,735 \pm 272 *$ & $1,617 \pm 329$ \\
\hline $72 \mathrm{~h}$ post ex & $2,018 \pm 477^{*}$ & $1,833 \pm 436$ & $2,074 \pm 487 *$ & $1,859 \pm 463$ & $1,868 \pm 291^{*}$ & $1,724 \pm 290$ \\
\hline \multicolumn{7}{|c|}{ Mid-thigh circumference $(\mathrm{cm})$} \\
\hline Baseline & $56.2 \pm 4.5$ & $56.1 \pm 4.5$ & $56.7 \pm 3.7$ & $56.6 \pm 3.4$ & $57.3 \pm 3.8$ & $57.4 \pm 3.7$ \\
\hline $0 \mathrm{~h}$ post ex & $56.8 \pm 4.6$ & $56.7 \pm 4.6$ & $57.4 \pm 3.8$ & $57.1 \pm 3.3$ & $57.8 \pm 3.8$ & $57.9 \pm 3.7$ \\
\hline $24 \mathrm{~h}$ post ex & $56.4 \pm 4.5^{*}$ & $56.9 \pm 4.7$ & $57.1 \pm 3.8^{*}$ & $57.6 \pm 3.2$ & $58.1 \pm 3.9$ & $58.1 \pm 3.8$ \\
\hline $48 \mathrm{~h}$ post ex & $56.3 \pm 4.6^{*}$ & $56.9 \pm 4.7$ & $56.9 \pm 3.8^{*}$ & $57.4 \pm 3.3$ & $57.9 \pm 3.9$ & $58.0 \pm 3.7$ \\
\hline $72 \mathrm{~h}$ post ex & $56.3 \pm 4.5^{*}$ & $56.7 \pm 4.7$ & $56.9 \pm 3.8^{*}$ & $57.1 \pm 3.3$ & $57.6 \pm 3.8$ & $57.8 \pm 3.8$ \\
\hline \multicolumn{7}{|c|}{ Creatine kinase (U/L) } \\
\hline Baseline & $176 \pm 76$ & $218 \pm 168$ & $223 \pm 222$ & $189 \pm 45$ & $199 \pm 241$ & $143 \pm 105$ \\
\hline $0 \mathrm{~h}$ post ex & $229 \pm 147$ & $245 \pm 220$ & $203 \pm 175$ & $193 \pm 156$ & $269 \pm 411$ & $165 \pm 105$ \\
\hline $24 \mathrm{~h}$ post ex & $736 \pm 1,115$ & $737 \pm 361$ & $231 \pm 182 *$ & $570 \pm 263$ & $312 \pm 242$ & $402 \pm 255$ \\
\hline $48 \mathrm{~h}$ post ex & $416 \pm 589$ & $361 \pm 318$ & $211 \pm 259$ & $263 \pm 174$ & $225 \pm 221^{*}$ & $748 \pm 1,694$ \\
\hline $72 \mathrm{~h}$ post ex & $359 \pm 433$ & $271 \pm 234$ & $204 \pm 343^{*}$ & $296 \pm 290$ & $151 \pm 57$ & $169 \pm 86$ \\
\hline \multicolumn{7}{|c|}{ Lactate dehydrogenase (U/L) } \\
\hline Baseline & $271 \pm 72$ & $218 \pm 107$ & $236 \pm 82$ & $207 \pm 61$ & $261 \pm 87$ & $256 \pm 93$ \\
\hline $0 \mathrm{~h}$ post ex & $280 \pm 87$ & $246 \pm 98$ & $227 \pm 95$ & $208 \pm 52$ & $278 \pm 85$ & $272 \pm 103$ \\
\hline $24 \mathrm{~h}$ post ex & $291 \pm 132$ & $270 \pm 123$ & $194 \pm 65$ & $194 \pm 69$ & $271 \pm 90$ & $269 \pm 97$ \\
\hline $48 \mathrm{~h}$ post ex & $264 \pm 117$ & $230 \pm 92$ & $177 \pm 71$ & $204 \pm 89$ & $260 \pm 69$ & $280 \pm 68$ \\
\hline $72 \mathrm{~h}$ post ex & $254 \pm 109$ & $247 \pm 112$ & $183 \pm 68$ & $219 \pm 75$ & $254 \pm 83$ & $267 \pm 77$ \\
\hline \multicolumn{7}{|c|}{ Myoglobin (ng/mL) } \\
\hline Baseline & $44.1 \pm 22.3$ & $47.8 \pm 38.4$ & $36.4 \pm 17.8$ & $27.2 \pm 7.71$ & $35.6 \pm 22.8$ & $27.3 \pm 7.7$ \\
\hline $0 \mathrm{~h}$ post ex & $95.4 \pm 76.6$ & $116.2 \pm 101.1$ & $60.7 \pm 30.1$ & $67.5 \pm 24.9$ & $65.1 \pm 44.3$ & $74.8 \pm 68.1$ \\
\hline $24 \mathrm{~h}$ post ex & $67.2 \pm 51.1$ & $69.5 \pm 54.9$ & $44.9 \pm 25.4$ & $38.5 \pm 13.3$ & $39.8 \pm 23.2$ & $47.3 \pm 22.7$ \\
\hline \multicolumn{7}{|c|}{ Interleukin-6 (pg/mL) } \\
\hline Baseline & $1.5 \pm 0.6$ & $1.7 \pm 0.7$ & $3.6 \pm 3.9$ & $2.6 \pm 2.3$ & $1.7 \pm 1.1$ & $2.6 \pm 2.7$ \\
\hline $0 \mathrm{~h}$ post ex & $2.2 \pm 0.7$ & $2.6 \pm 1.1$ & $4.5 \pm 6.8$ & $3.4 \pm 3.2$ & $2.3 \pm 1.3$ & $2.7 \pm 2.8$ \\
\hline $24 \mathrm{~h}$ post ex & $1.5 \pm 0.9$ & $1.9 \pm 1.0$ & $3.7 \pm 6.3$ & $2.8 \pm 2.5$ & $1.7 \pm 0.9$ & $2.4 \pm 2.1$ \\
\hline
\end{tabular}

Appropriate statistics were completed using log transformed values $* P<0.05$ 